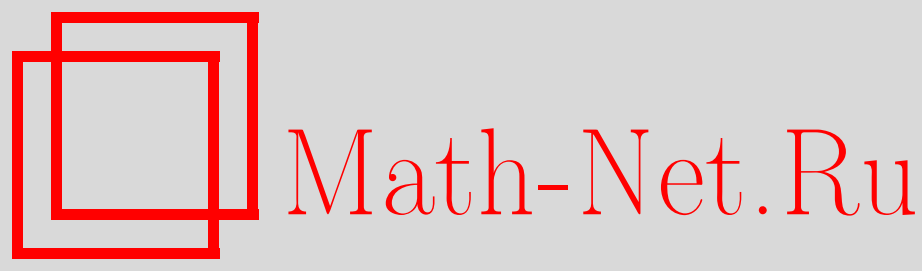

А. А. Толченников, Простые точные решения и асимптотика локализованных решений двумерного безмассового уравнения Дирака для графена, ТМФ, 2018, том 196, номер 1, 64-69

DOI: https://doi.org/10.4213/tmf9521

Использование Общероссийского математического портала Math-Net.Ru подразумевает, что вы прочитали и согласны с пользовательским соглашением http://www . mathnet.ru/rus/agreement

Параметры загрузки:

IP : 54.210 .77 .194

26 апреля 2023 г., 17:38:01

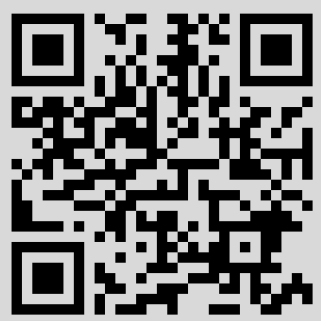




\title{
ПРОСТЫЕ ТОЧНЫЕ РЕШЕНИЯ И АСИМПТОТИКА ЛОКАЛИЗОВАННЫХ РЕШЕНИЙ ДВУМЕРНОГО БЕЗМАССОВОГО УРАВНЕНИЯ ДИРАКА ДЛЯ ГРАФЕНА
}

\begin{abstract}
Построены точные решения и асимптотика локализованных решений двумерного безмассового уравнения Дирака для графена с малым потенциалом.
\end{abstract}

Ключевые слова: безмассовое уравнение Дирака для графена, точные решения, операторы рождения.

DOI: https://doi.org/10.4213/tmf9521

\section{1. ВВЕДЕНИЕ}

В статье [1] было получено богатое семейство точных алгебраических решений многомерного волнового уравнения. По существу эти решения являются обобщениями решений, рассмотренных в работах [2]. Для двумерного волнового уравнения с постоянной скоростью $c$

$$
\frac{\partial^{2} u}{\partial t^{2}}-c^{2}\left(\frac{\partial^{2} u}{\partial x_{1}^{2}}+\frac{\partial^{2} u}{\partial x_{2}^{2}}\right)=0
$$

такие (комплексные) решения имеют вид

$$
u(x, t)=A \frac{1+i c t / \mu}{[(1+i(c t+|x|) / \mu)(1+i(c t-|x|) / \mu)]^{3 / 2}},
$$

где $A$ - произвольная комплексная константа. Вещественная (и мнимая) часть этой функции также есть решение исходного волнового уравнения. Если $A$ вещественно,

Исследование выполнено за счет гранта РНФ (проект № 16-11-10282).

${ }^{*}$ Институт проблем механики им. А. Ю. Ишлинского РАН, Москва, Россия. E-mail: tolchennikovaa@gmail.com

${ }^{\dagger}$ Московский физико-технический институт (государственный университет), Долгопрудный, Московская обл., Россия 
то $\left.\operatorname{Im} u\right|_{t=0}=0$. Параметр $\mu$ здесь является произвольным, хотя в дальнейшем мы будем считать его положительным и достаточно малым. Параметр $\mu$ определяет "размер" локализации начальной волновой функции. Структура решения $u$ имеет следующий вид: при $t=0$ оно сосредоточено в окрестности точки $x=0$, а через какое-то время становится сосредоточенным в окрестности окружности $|x|=c t$. Эта окружность есть фронт решения, он образован концами траекторий $x=c t \mathbf{n}(\varphi)$, где $\varphi$ - полярный угол на плоскости $\left(x_{1}, x_{2}\right), \mathbf{n}(\varphi)=\left(\begin{array}{c}\cos \varphi \\ \sin \varphi\end{array}\right)$. Если рассмотреть это решение в какой-то момент времени $t>0$ и затем рассмотреть его эволюцию в обратном времени, то мы получаем, что траектории, выпущенные из окружности, фокусируются в одной точке $x=0$, которую, тем самым, можно считать фокальной точкой.

Дифференцирование решения $u$ по $x_{1}, x_{2}, t$ дает новые решения волнового уравнения, поэтому об операторах $\partial / \partial x_{j}, \partial / \partial t$ можно говорить как об аналогах "операторов рождения" в квантовой механике. Получаемые таким образом решения по-прежнему локализованы в окрестности фронта $|x|=c t$, однако, во-первых, профиль решения в окрестности фронта может иметь несколько осцилляций и, во-вторых, профиль зависит от точки (угла $\varphi$ ) фронта, а в каких-то точках фронта решение может вообще обращаться в ноль.

Далее мы показываем, что решения такого же типа существуют и у безмассовой двумерной системы уравнений Дирака, описывающей эволюцию волновых функций в графене (см. книгу [3]):

$$
\begin{aligned}
& i \frac{\partial u}{\partial t}=\left(-i \frac{\partial}{\partial x_{1}}+\frac{\partial}{\partial x_{2}}\right) v+U(x) u \\
& i \frac{\partial v}{\partial t}=\left(-i \frac{\partial}{\partial x_{1}}-\frac{\partial}{\partial x_{2}}\right) u+U(x) v .
\end{aligned}
$$

\section{2. ТОЧНЫЕ РЕШЕНИЯ В СЛУЧАЕ ПОСТОЯННОГО ПОТЕНЦИАЛА}

Пусть сначала потенциал $U$ равен константе. Тогда $w_{1}=e^{i U t} u$ и $w_{2}=e^{i U t} v$ и их комплексно-сопряженные функции являются решениями волнового уравнения (1). Выбирая $w_{1}$ и $w_{2}$ в виде $(2)$ и восстанавливая вторые компоненты этих решений, приходим к формулам для решений вида

$$
\begin{gathered}
\left(\begin{array}{l}
u(x, t) \\
v(x, t)
\end{array}\right)=e^{-i U t}\left(\alpha_{1} \psi_{1}(x, t)+\alpha_{2} \psi_{2}(x, t)\right), \\
\psi_{1}=\frac{1}{\left((1+i t / \mu)^{2}+|x|^{2} / \mu^{2}\right)^{3 / 2}}\left(\begin{array}{c}
i\left(x_{1}+i x_{2}\right) / \mu \\
1+i t / \mu
\end{array}\right), \\
\psi_{2}=\frac{1}{\left((1-i t / \mu)^{2}+|x|^{2} / \mu^{2}\right)^{3 / 2}}\left(\begin{array}{c}
1-i t / \mu \\
-i\left(x_{1}-i x_{2}\right) / \mu
\end{array}\right),
\end{gathered}
$$

где $\alpha_{1}, \alpha_{2}$ - произвольные комплексные константы. Дифференцируя эти решения по $x_{1}, x_{2}, t$, мы получаем новые решения. 


\section{3. АСИМПТОТИКА ЛОКАЛИЗОВАННЫХ РЕШЕНИЙ В СЛУЧАЕ ПЕРЕМЕННОГО ПОТЕНЦИАЛА}

В случае переменного потенциала едва ли возможно найти точные решения, но можно найти асимптотику решения по малому параметру $\mu$ - характерной ширине начального условия для системы (3). А именно, рассмотрим задачу Коши для системы уравнений (3) с начальными условиями

$$
\left.u\right|_{t=0}=u^{0}\left(\frac{x}{\mu}\right),\left.\quad v\right|_{t=0}=v^{0}\left(\frac{x}{\mu}\right),
$$

где $u^{0}(z), v^{0}(z)$ - гладкие функции, достаточно быстро убывающие при $|z| \rightarrow \infty$. А именно, потребуем, чтобы они убывали быстрее, чем const $|z|^{-\beta}(\beta>2)$, и их производные $\partial^{|\alpha|} / \partial z^{\alpha}$ (где $\left.\alpha=\left(\alpha_{1}, \alpha_{2}\right),|\alpha|=\alpha_{1}+\alpha_{2}\right)$ убывали на бесконечности быстрее const $|z|^{-\beta-|\alpha|}$. Также предполагаем, что гладкий потенциал $U(x)$ постоянен вне некоторого компакта. Решение задачи Коши ищется на конечном интервале времени $[0, T]$.

Воспользуемся результатами работ [4], [5], в которых были построены асимптотики решения для строго гиперболических систем уравнений с локализованными начальными условиями вида $V(x / \mu)$.

При $t=0$ решение локализовано в окрестности точки $x=0$, а в следующие моменты времени решение оказывается локализовано в окрестности окружности (фронта) $|x|=t$. Приведем формулу для асимптотики решения в окрестности фронта.

Tеорема 1. При $t>t_{0}>0$, в некоторой не зависящей от $\mu$ окрестности фронта $|x|=t$ асимптотика при $\mu \rightarrow 0$ решения задачи (3) с начальным условием (5) имеет вид

$$
\Psi=\left(\begin{array}{l}
u \\
v
\end{array}\right)=\frac{1}{2} \sqrt{\frac{\mu}{2 \pi t}} \exp \left[-i \int_{0}^{t} U\left(\frac{\tau x}{|x|}\right) d \tau\right]\left(f^{+}\left(x_{1}, x_{2}, t, \mu\right)+f^{-}\left(x_{1}, x_{2}, t, \mu\right)+o(1)\right),
$$

где

$$
\begin{gathered}
f^{ \pm}\left(x_{1}, x_{2}, t, \mu\right)=e^{\mp i \pi / 4} \int_{0}^{\infty} \sqrt{\rho} e^{ \pm i \rho(|x|-t) / \mu}\left(\begin{array}{cc}
1 & \frac{x_{1}+i x_{2}}{|x|} \\
\frac{x_{1}-i x_{2}}{|x|} & 1
\end{array}\right)\left(\begin{array}{c}
\tilde{u}^{0}\left( \pm \frac{\rho x}{|x|}\right) \\
\tilde{v}^{0}\left( \pm \frac{\rho x}{|x|}\right)
\end{array}\right) d \rho, \\
\tilde{u}^{0}(p)=\frac{1}{2 \pi} \int_{\mathbb{R}^{2}} e^{-i p x} u^{0}(x) d x, \quad \tilde{v}^{0}(p)=\frac{1}{2 \pi} \int_{\mathbb{R}^{2}} e^{-i p x} v^{0}(x) d x .
\end{gathered}
$$

3.1. “Операторы рождения”. Пусть $\Psi$ и $\Psi_{Q}$ - решения задачи (3) с начальными условиями $\left(\begin{array}{c}u^{0}(x / \mu) \\ v^{0}(x / \mu)\end{array}\right)$ и $Q\left(\mu \frac{\partial}{\partial x_{1}}, \mu \frac{\partial}{\partial x_{2}}\right)\left(\begin{array}{c}u^{0}(x / \mu) \\ v^{0}(x / \mu)\end{array}\right)$ соответственно, где $Q\left(p_{1}, p_{2}\right)$ есть многочлен от $p_{1}, p_{2}$. Тогда из теоремы 1 получается, что в окрестности фронта $|x|=t$ (при $\left.t>t_{0}>0\right)$

$$
\Psi_{Q} \approx Q\left(\mu \frac{\partial}{\partial x_{1}}, \mu \frac{\partial}{\partial x_{2}}\right) \Psi \approx Q\left(-\mu \frac{x_{1}}{|x|} \frac{\partial}{\partial t},-\mu \frac{x_{2}}{|x|} \frac{\partial}{\partial t}\right) \Psi,
$$

где знак $\approx$ между функциями означает, что эти функции имеют одинаковый старший член асимптотики по $\mu$. 
В этом смысле операторы $Q\left(\mu \frac{\partial}{\partial x_{1}}, \mu \frac{\partial}{\partial x_{2}}\right), Q\left(-\mu \frac{x_{1}}{|x|} \frac{\partial}{\partial t},-\mu \frac{x_{2}}{|x|} \frac{\partial}{\partial t}\right)$ являются операторами рождения: их применение к уже известному решению дает функцию, которая имеет тот же старший член асимптотики, что и некоторое новое решение (аналогичные операторы для системы мелкой воды были построены в работе [6]).

3.2. Начальные условия специального вида. Рассмотрим функцию $f(x)=$ $g(T(\theta) x)$, где

$$
g(z)=\left(1+\left(\frac{z_{1}}{b}\right)^{2}+\left(\frac{z_{2}}{c}\right)^{2}\right)^{-3 / 2}, \quad T(\theta)=\left(\begin{array}{rr}
\cos \theta & \sin \theta \\
-\sin \theta & \cos \theta
\end{array}\right),
$$

$b, c, \theta$ - действительные параметры. Преобразование Фурье функции $g(z)$ имеет простой вид $\tilde{g}\left(p_{1}, p_{2}\right)=b c e^{-|p| \beta(\psi)}$, где $\beta(\psi)=\sqrt{b^{2} \cos ^{2} \psi+c^{2} \sin ^{2} \psi}$. Поэтому $\tilde{f}(\rho \mathbf{n}(\psi))=b c e^{-\rho \beta(\psi-\theta)}$ (см. статью [7]). Рассмотрим следующие функции, задающие начальные условия (5):

$$
\begin{aligned}
& u^{0}(y)=a_{1}\left[1+\left(\frac{\cos \theta_{1} y_{1}+\sin \theta_{1} y_{2}}{b_{1}}\right)^{2}+\left(\frac{-\sin \theta_{1} y_{1}+\cos \theta_{1} y_{2}}{c_{1}}\right)^{2}\right]^{-3 / 2}, \\
& v^{0}(y)=a_{2}\left[1+\left(\frac{\cos \theta_{2} y_{1}+\sin \theta_{2} y_{2}}{b_{2}}\right)^{2}+\left(\frac{-\sin \theta_{2} y_{1}+\cos \theta_{2} y_{2}}{c_{2}}\right)^{2}\right]^{-3 / 2} .
\end{aligned}
$$

Тогда функции $f^{ \pm}$из теоремы 1 принимают вид

$$
\begin{aligned}
f^{ \pm}\left(x_{1}, x_{2}, t, \mu\right)= & e^{\mp i \pi / 4} a_{1} b_{1} c_{1} \frac{\sqrt{\pi}}{2}\left(\frac{|x|}{x_{1}+i x_{2}}\right)\left(\beta\left(\arg x-\theta_{1}\right) \mp \frac{i}{\mu}(|x|-t)\right)^{-3 / 2}+ \\
& +e^{\mp i \pi / 4} a_{2} b_{2} c_{2} \frac{\sqrt{\pi}}{2}\left(\begin{array}{c}
\frac{x_{1}+i x_{2}}{|x|} \\
1
\end{array}\right)\left(\beta\left(\arg x-\theta_{2}\right) \mp \frac{i}{\mu}(|x|-t)\right)^{-3 / 2} .
\end{aligned}
$$

3.3. Доказательство теоремы 1. Доказательство заключается в применении алгоритма, изложенного в статье [4] (а также фактически в работах [7], [8]). Систему (3) и начальные условия (5) перепишем в виде

$$
i \frac{\partial \Psi}{\partial t}=A\left(x,-i \frac{\partial}{\partial x}\right) \Psi,\left.\quad \Psi\right|_{t=0}=V\left(\frac{x}{\mu}\right), \quad \text { где } \quad A(x, p)=\left(\begin{array}{cc}
U(x) & p_{1}+i p_{2} \\
p_{1}-i p_{2} & U(x)
\end{array}\right) .
$$

Старшая однородная часть первого порядка матричного гамильтониана имеет вид $A_{0}=\left(\begin{array}{cc}0 & p_{1}+i p_{2} \\ p_{1}-i p_{2} & 0\end{array}\right)$. Собственные значения $A_{0}$ записываются как $H^{ \pm}= \pm|p|$.

Применим последовательно шаги алгоритма из статьи [4].

1. Из окружности $\Gamma_{0}=\left\{p=\mathbf{n}(\psi)=\left(\begin{array}{c}\cos \psi \\ \sin \psi\end{array}\right), x=0\right\}$ выпускаем траектории гамильтоновых систем

$$
\dot{x}=\frac{\partial H^{ \pm}}{\partial p}, \quad \dot{p}=\frac{\partial H^{ \pm}}{\partial x} .
$$

Решениями этих систем являются функции $X^{ \pm}(t, \psi)= \pm t \mathbf{n}(\psi), P^{ \pm}(\psi)=\mathbf{n}(\psi)$. При фиксированном $t$ функции $X^{ \pm}(t, \psi), P^{ \pm}(\psi)$ задают параметризацию волнового фронта $\Gamma_{t}^{ \pm}$в фазовом пространстве, а проекции на конфигурационное пространство дают окружность $|x|=t$. Решение нашей задачи при $t>0$ оказывается локализованным в окрестности этой окружности. 


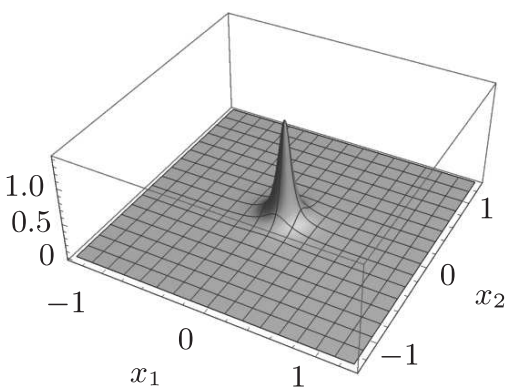

a

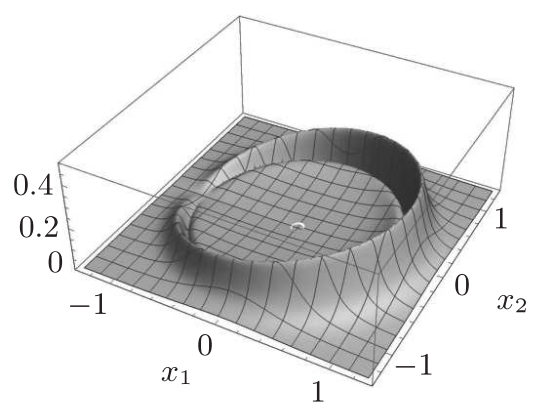

б

Рис. 1. Графики старшего члена асимптотики $|\Psi|$ для $t=0$ (а) и $t=1$ (б).

2. Находим $w^{ \pm}(p)$ - нормированные собственные векторы матрицы $A_{0}$ :

$$
w^{ \pm}(p)=\frac{1}{\sqrt{2}}\left(\begin{array}{c} 
\pm 1 \\
\frac{p_{1}-i p_{2}}{|p|}
\end{array}\right) .
$$

3. Решаем уравнения переноса и находим функции $M^{ \pm}(t, \psi)=-i \int_{0}^{t} U( \pm \tau \mathbf{n}(\psi)) d \tau$.

4. Находим $c^{ \pm}(\rho, \psi)$ - коэффициенты разложения $\widetilde{V}(\rho \mathbf{n}(\psi))$ (где $\widetilde{V}$ - преобразование Фурье $V(x))$ по базису $w^{ \pm}$, вычисленному при $p=\rho \mathbf{n}(\psi)$ :

$$
\left(\begin{array}{l}
c^{+}(\rho, \psi) \\
c^{-}(\rho, \psi)
\end{array}\right)=\frac{1}{\sqrt{2}}\left(\begin{array}{cc}
1 & e^{i \psi} \\
-1 & e^{i \psi}
\end{array}\right) \widetilde{V}(\rho \mathbf{n}(\psi)) .
$$

5. Находим индексы Маслова для траекторий $\left(X^{ \pm}(t, \psi), P^{ \pm}(\psi)\right): m^{+}(t, \psi) \equiv 0$, $m^{-}(t, \psi) \equiv-1$.

6. Находим длины векторов $V_{1}=\left|n_{\psi}\right|=1, V_{2}=\left|X_{\psi}^{ \pm}\right|=t$.

Согласно теореме 1 из работы [4] в некоторой не зависящей от $\mu$ окрестности окружности $|x|=t>t_{0}$ асимптотика решения имеет вид

$$
\begin{array}{r}
\sum_{\sigma \in\{+,-\}} \sqrt{\frac{\mu}{2 \pi}} e^{-i \pi / 4-i \pi m^{\sigma} / 2} \int_{0}^{\infty} \sqrt{\rho} e^{i \rho\left\langle P^{\sigma}(t, \psi), x-X^{\sigma}(t, \psi)\right\rangle / \mu} \times \\
\times\left.\sqrt{\frac{\left|P^{\sigma}(t, \psi)\right| V_{1}}{V_{2}}}\left(c^{\sigma} e^{M^{\sigma}} w^{\sigma}+o(1)\right)\right|_{\psi=\psi^{\sigma}(t, x)} d \rho,
\end{array}
$$

где $\psi^{+}(t, x)=\arg x, \psi^{-}(t, x)=\arg x+\pi$. Подставляя в эту формулу все найденные величины, получаем формулу (6).

\section{4. ПРИМЕР}

Пусть потенциал $U\left(x_{1}, x_{2}\right)$ равен $x_{1}$ в некотором достаточно большом компакте и равен константе вне некоторого компакта. Приведем несколько рисунков, иллюстрирующих поведение модуля решения $\Psi=(u, v)$ при значении параметра $\mu=0.2$. Выберем функции, задающие начальное условие, в специальном виде $u^{0}(y)=v^{0}(y)=\left(1+|y|^{2}\right)^{-3 / 2}$. Графики старшего члена асимптотики функции $|\Psi|=\sqrt{|u|^{2}+|v|^{2}}$ для $t=0$ и $t=1$ показаны на рис. 1 . 


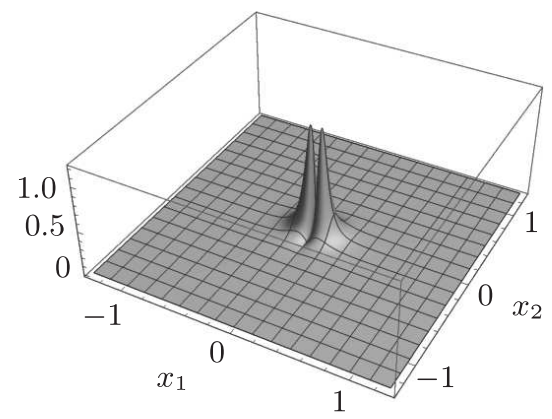

a

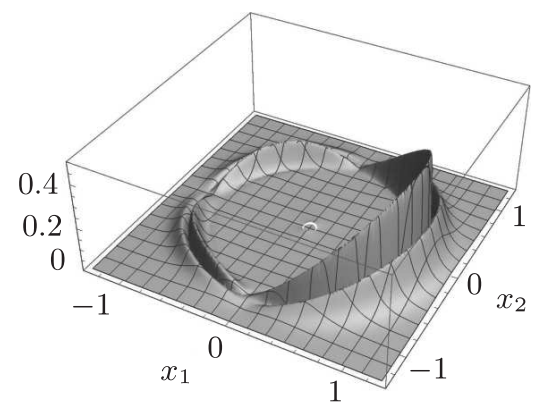

б

Рис. 2. Графики старшего члена асимптотики $\left|\Psi_{Q}\right|$ после применения "оператора рождения" $\hat{Q}=\mu \frac{\partial}{\partial x_{1}}$ при $t=0$ (а) и $t=1$ (б).

Теперь применим “оператор рождения" $\hat{Q}=\mu \frac{\partial}{\partial x_{1}}$. Соответствующие графики старшего члена асимптотики функции $\left|\Psi_{Q}\right|$ для $t=0$ и $t=1$ приведены на рис. 2.

Благодарности. Автор благодарен С. Ю. Доброхотову за постановку задачи и дискуссии, а также В. Е. Назайкинскому за ценные замечания.

\section{Список литературы}

[1] С. Ю. Доброхотов, С. Я. Секерж-Зенькович, "Один класс точных алгебраических локализованных решений многомерного волнового уравнения", Матем. заметки, 88:6 (2010), 942-945.

[2] Л. Н. Сретенский, Теория волновых движений жидкости, Наука, М., 1977; С. Ф. Доценко, Б. Ю. Сергиевский, Л.В. Черкасов, "Пространственные волны цунами, вызванные знакопеременным смещением поверхности океана", Исследования иунами, Вып. 1, Междувед. геофиз. ком., M., 1986, 7-14; S. Wang, B. Le Mehaute, C.-C. Lu, "Effect of dispersion on impulsive waves", Mar. Geophys. Res., 9:2 (1987), 95-111; S. Ya. Sekerzh-Zenkovich, "Simple asymptotic solution of the Cauchy-Poisson problem for head waves", Russ. J. Math. Phys., 16:2 (2009), 315-322.

[3] M.I. Katsnelson, Graphene: Carbon in Two Dimensions, Cambridge Univ. Press, New York, 2012.

[4] А. И. Аллилуева, С. Ю. Доброхотов, С. А. Сергеев, А. И. Шафаревич, "Новые представления канонического оператора Маслова и локализованные асимптотические решения строго гиперболических систем", Докл. РАН, 464:3 (2015), 261-266.

[5] С. Ю. Доброхотов, П. Н. Жевандров, В. П. Маслов, А. И. Шафаревич, “Асимптотические быстроубывающие решения линейных строго гиперболических систем с переменными коэффициентами", Матем. заметки, 49:4 (1991), 31-46.

[6] С. А. Сергеев, А. А. Толченников, "Об 'операторах рождения' в задаче о локализованных решениях линеаризованных уравнений мелкой воды с регулярными и особыми характеристиками", Матем. заметки, 100:6 (2016), 911-922.

[7] S. Yu. Dobrokhotov, R. V. Nekrasov, B. Tirozzi, "Asymptotic solutions of the linear shallow-water equations with localized initial data", J. Eng. Math., 69:2-3 (2011), 225-242.

[8] S. Yu. Dobrokhotov, A. I. Shafarevich, B. Tirozzi, "Localized wave and vortical solutions to linear hyperbolic systems and their application to linear shallow water equations", Russ. J. Math. Phys., 15:2 (2008), 192-221. 